\title{
Vortex State Suppression in a Hexagonal Array of Interacting Py Triangular Rings
}

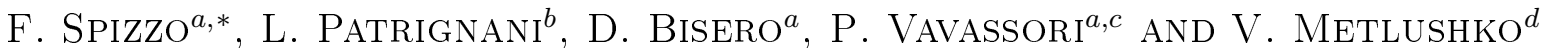 \\ ${ }^{a}$ CNISM \& Department of Physics and Earth Sciences, University of Ferrara, via G. Saragat 1, I-44122 Italy \\ ${ }^{b}$ Imperial College of London, Blackett Laboratory, London SW7 2BW, UK \\ ${ }^{c}$ CIC nanoGUNE Consolider, Tolosa Hiribidea 76, E-20018 Donostia-San Sebastián, Spain \\ ${ }^{d}$ Dept. of Electrical and Computer Eng., University of Illinois, Chicago, Illinois 60612, USA
}

\begin{abstract}
We investigate the effect of magnetostatic interactions on the magnetization reversal process of equilateral triangular rings arranged on a hexagonal lattice. Inter-ring interactions originate as rings corners host domain-walls that produce an intense dipolar field; the effect of such interactions is studied by recording hysteresis loops for different magnetic field orientations. Rings magnetic configuration is probed via magnetic force microscopy and diffraction magneto-optic Kerr effect measurements. We observe that the effect of stray fields due to domain-walls competes with that of shape anisotropy, and that the former prevails when the magnetic field orientation is parallel to rings symmetry axes.
\end{abstract}

DOI: 10.12693/APhysPolA.123.218

PACS: 75.60.Jk, 75.75.Fk, 75.30.Gw

\section{Introduction}

Magnetic nanorings (MNs) made of soft magnetic materials have been recently the subject of intense investigation as their magnetic configuration during reversal can be largely controlled thanks to their geometry [1]. On the one hand, great attention is devoted to MNs possible applications as data storage materials, particularly in magnetic-RAM (MRAM) cells, and magnetic sensors. On the other hand, the interest in MNs is due to the understanding of their basic physical properties. Eventually, as their magnetization reversal process takes place through domain-wall (DW) creation and annihilation, if nanorings distance is small, DW interactions may be investigated [2].

When triangular rings are considered, during magnetization reversal their magnetic configuration goes through the so called vortex state (VS) and onion state (OS) [3]. In the former, the MN displays a continuous magnetization profile producing a weak stray field (WSF) close to each corner, thus the ring has three magnetically equivalent WSF corners. In the OS, two out of three corners host a DW, thus producing an intense stray field (ISF), whilst the third corner is of WSF type. Due to that, when the MNs interdistance is small, the stray fields produced by ISF corners may influence the behavior of the DWs in the adjacent rings and affect rings switching process, accordingly. In an earlier work, we studied the effects of dipolar interactions between two adjacent corners, viz. two DWs [3], and we found that interactions induced a change in switching fields values. In this paper, we investigate a different configuration, namely the rings are ordered in a hexagonal pattern (see Fig. 1a); with this

*corresponding author; e-mail: spizzo@fe.infn.it
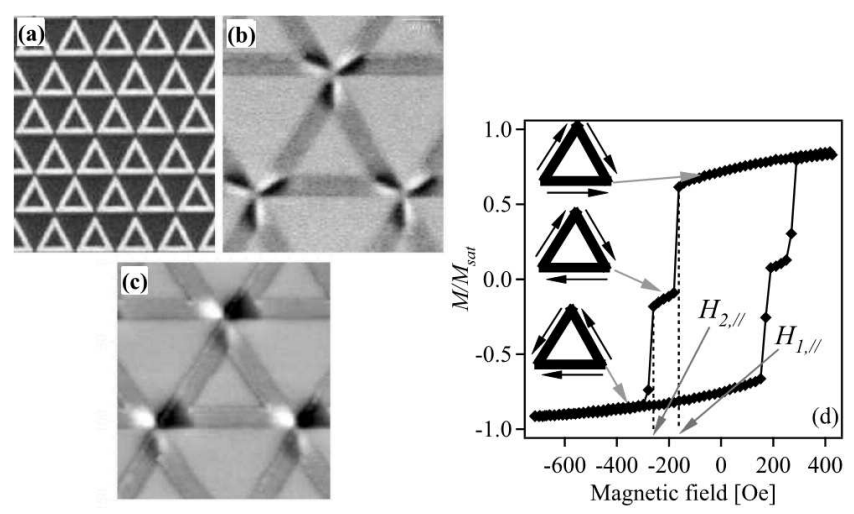

Fig. 1. (a) SEM image of the array of triangular rings arranged on a triangular lattice; the side of each ring has a nominal size of $2 \mu \mathrm{m}$. MFM detail of the VS (b), where three equivalent WSF corners can be observed, and of the OS (c), with two ISF and one WSF corner. In this regard, MFM images show that, at a given $H$, all the rings display the same magnetic configuration; this result may feature the presence of a strong inter-ring coupling. (d) Magnetization loop of the MNs ordered on a hexagonal lattice recorded in $H_{\|}$configuration; $H_{1, \|}$ and $H_{2, \|}$ represent the first and the second switching field, respectively. The arrows close to the equilateral triangles represent the orientation of the magnetization in each side of the ring at the specified position of the loop.

geometry, three adjacent corners are found and the effect of inter-ring interactions is expected to be stronger.

\section{Experimental}

The permalloy (Py) equilateral triangular rings have nominal side length of $1.8 \mu \mathrm{m}$, side width of $230 \mathrm{~nm}$, and 
thickness of $25 \mathrm{~nm}$. The rings are ordered on a hexagonal lattice (see Fig. 1a), and the corner-to-corner distance is $50 \mathrm{~nm}$. The ring structures have been lithographically patterned on a $25 \mathrm{~nm}$ thick Py film deposited onto a standard Si substrate followed by the lift-off step. Magnetization $(M)$ reversal was studied performing magneto-optic Kerr effect (MOKE) measurements, with an in-plane magnetic field $(H)$, in longitudinal and diffraction configuration [4], L-MOKE and D-MOKE, respectively. The latter allows to map the magnetic form factor of the MN, thus supplying details about the spatial symmetry of the MN magnetic configuration. More details about L- and D-MOKE can be found in [3-5]. The MNs were also investigated with a Digital Instruments Nanoscope IIIa magnetic force microscope (MFM).

\section{Results and discussion}

The magnetization loop recorded for $H$ parallel $\left(H_{\|}\right)$ to an edge of the triangular rings is presented in Fig. $1 \mathrm{~d}$. Starting from saturation, as $H$ is reduced the switching of the base of the ring is first observed at $H_{1, \|}$ so the MN moves from the OS to the VS (see Fig. 1d); then the two sides switch at $H_{2, \|}$ and the MN moves to the opposite OS. The VS and OS MFM maps are presented in Fig. 1b and c. On the reverse branch of the loop, the same OSs are found, whilst the VS changes chirality. This result is not apparent from Fig. 1d and can be better appreciated looking at the first order D-MOKE hysteresis loop (Fig. 2a).
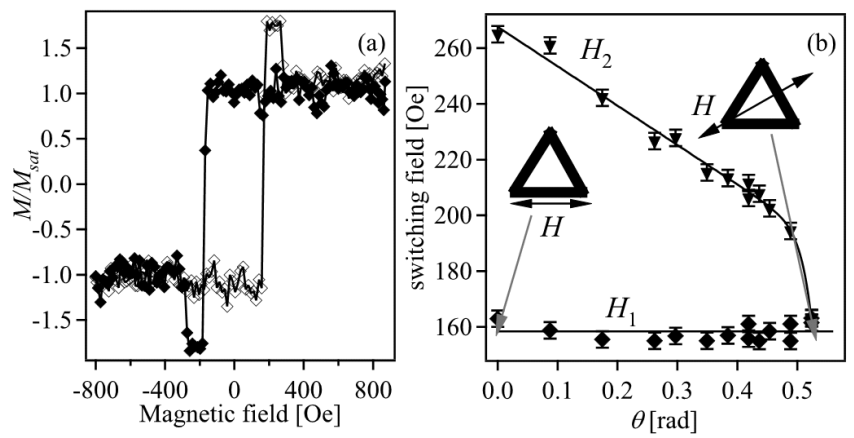

Fig. 2. (a) First order D-MOKE loop recorded in $H_{\|}$ configuration: first $(\diamond)$ and reverse $(\diamond)$ branch. (b) $H_{1}$ and $H_{2}$ dependence on $H$ orientation; straight lines are just a guide to the eye.

Indeed, the downward peak observed in the first branch of the loop in correspondence of the VS is turned to upward in the reverse branch due to the change of VS chirality. In the $H_{\|}$configuration, the measured $H_{1, \|}$ and $H_{2, \|}$ values are smaller than those recorded for non-interacting $\mathrm{MNs}$ of $\approx 20 \mathrm{Oe}$ and $\approx 80 \mathrm{Oe}$, respectively [3]. Beyond that, the sequence of states explored by the MNs during the reversal process is not affected by magnetostatic interactions. Owing to the symmetry of the lattice, the same behavior is observed under $\pi / 3$ rotations of the external field ${ }^{\dagger}$. If $H$ is rotated by $\pi / 6$, i.e. it becomes perpendicular to an edge $\left(H_{\perp}\right)$, we find that $H_{1, \perp}=H_{2, \perp}$, so the VS turns out to be unstable and the MNs switch directly from one OS to the next; in particular, $H_{1, \perp}=H_{1, \|}$. By comparison, in non-interacting MNs the VS was observed in the $H_{\perp}$ configuration: the first switching field value was close to $H_{1, \perp}$ whilst the second was 80 Oe larger. In addition, the $H$ range of existence of the VS in the $H_{\perp}$ configuration was about one half of that measured in the $H_{\|}$configuration.

To study what affects the stability of the VS, we investigated the effect of $H$ orientation on the magnetization reversal process. If $\theta$ is defined as the angle between the $H$ direction and the direction parallel to one edge, we recorded the $H_{1}$ and $H_{2}$ values as a function of $\theta$ (see Fig. 2b). The $H_{1}$ value is nearly independent of $\theta$; the $H_{2}$ value linearly decreases with $\theta$ and then drops suddenly to the $H_{1}$ value when $\theta \approx \pi / 6$. Therefore, the VS is unstable only in a narrow angular range. Conversely, for the non-interacting MNs both $H_{1}$ and $H_{2}$ had a linear dependence on $\theta$ and $H_{2}$ was always larger than $H_{1}$. This difference may be explained in terms of competition between shape anisotropy and dipolar interaction. In the $H_{\|}$configuration, the OS $\rightarrow$ VS switch is favored by $H$ and by a reduction of magnetostatic energy, as thanks to that switch the ISF corners disappear. After that, the VS remains stable due to shape anisotropy; indeed, the OS $\rightarrow$ VS transition is produced by the switching of the base of the ring at $H_{1, \|}$ and the sides do not switch at the same field as they are not parallel to $H$. So the second switch, VS $\rightarrow$ OS, takes place at a higher value of applied field $\left(\mathrm{H}_{2, \|}\right)$.

In $H_{\perp}$ configuration the $\mathrm{OS} \rightarrow \mathrm{VS}$ switch is favored by the same contributions and, in absence of interactions, the VS is stabilized by a reduction of the magnetostatic energy contribution and not by the shape anisotropy term. Indeed, the OS $\rightarrow$ VS transition is produced by the switch of one of the two sides of the ring, that in this case are equivalent with each other as the field is applied along a symmetry axis for the ring, so in principle both of them could rotate and form an OS. If interactions are considered, that reduction of magnetostatic energy in the VS may result smaller than the energy reduction that would be obtained in the OS, where two adjacent DWs, namely two adjacent ISF corners, strongly interact with each other (they produce magnetic charges having opposite sign, so flux closure can be achieved [2]). This is substantiated by the fact that, in the $H_{\|}$configuration, the VS $\rightarrow$ OS switch of the interacting MNs array is anticipated of $\approx 80$ Oe with respect to the non-interacting MNs. Therefore, in $H_{\perp}$ configuration, due to the effects

${ }^{\dagger} \mathrm{MN}$ shape is invariant under $2 \pi / 3$ rotations whilst the triangular lattice is invariant under $\pi / 3$ rotations; so, the L-MOKE and D-MOKE loops are invariant under $\pi / 3$ and $2 \pi / 3 H$ rotations, respectively 
of DW dipolar field, at $H_{2, \perp}$ the MNs switch directly from one OS to the next one.

\section{Conclusions}

We studied the effect of magnetostatic interactions on an array of triangular rings ordered on a hexagonal pattern. We found that DW interactions on average hamper the formation of the VS, that disappears when the magnetic field orientation is close to rings symmetry axis. In this case, the VS is unstable due to the lack of the shape anisotropy stabilizing effect, and DW interaction energy favors the OS appearance.

\section{Acknowledgments}

This work was supported by the European Community's Seventh Framework Programme (FP7/20072013) under grant agreement no. 228673 (MAGNONICS). The support given by MIUR under project FIRB 2010-NANOREST is also acknowledged.

\section{References}

[1] P. Vavassori, O. Donzelli, M. Grimsditch, V. Metlushko, B. Ilic, J. Appl. Phys. 101, 023902 (2007) M. Kläui, C.A.F. Vaz, J.A.C. Bland, L.J. Heyderman, Appl. Phys. Lett. 86, 032504 (2005)

[2] T.J. Hayward, M.T. Bryan, P.W. Fry, P.M. Fundi, M.R.J. Gibbs, D.A. Allwood, M.-Y. Im, P. Fischer, Phys. Rev. B 81, 020410 (2010); M. Klaüi, J. Phys., Condens. Matter. 20, 313001 (2008)

[3] P. Vavassori, D. Bisero, V. Bonanni, A. Busato, M. Grimsditch, K.M. Lebecki, V. Metlushko, B. Ilic, Phys. Rev. B 78, 174403 (2008)

[4] M. Grimsditch, P. Vavassori, J. Phys., Condens. Matter 16, R275 (2004).

[5] T. Verduci, C. Rufo, A. Berger, V. Metlushko, B. Ilic, P. Vavassori, Appl. Phys. Lett. 99, 095201 (2011) 